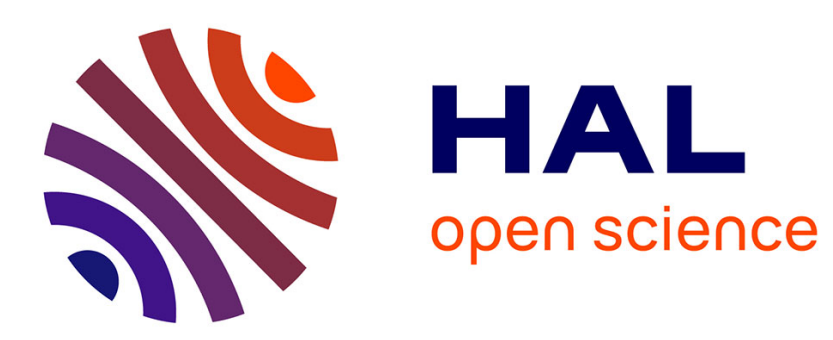

\title{
Reconnaissance de signaux acoustiques à l'aide d'un réseau neuro-mimétique multicouche
}

\author{
I. Coustaux, J. Trouilhet, J. Guilhot
}

\section{To cite this version:}

I. Coustaux, J. Trouilhet, J. Guilhot. Reconnaissance de signaux acoustiques à l'aide d'un réseau neuro-mimétique multicouche. Journal de Physique IV Proceedings, 1994, 04 (C5), pp.C5-1319-C51322. 10.1051/jp4:19945293 . jpa-00253006

\section{HAL Id: jpa-00253006 https://hal.science/jpa-00253006}

Submitted on 1 Jan 1994

HAL is a multi-disciplinary open access archive for the deposit and dissemination of scientific research documents, whether they are published or not. The documents may come from teaching and research institutions in France or abroad, or from public or private research centers.
L'archive ouverte pluridisciplinaire HAL, est destinée au dépôt et à la diffusion de documents scientifiques de niveau recherche, publiés ou non, émanant des établissements d'enseignement et de recherche français ou étrangers, des laboratoires publics ou privés. 


\title{
Reconnaissance de signaux acoustiques à l'aide d'un réseau neuro-mimétique multicouche
}

\author{
I. COUSTAUX, J.F. TROUILHET E J.P. GUILHOT
}

Laboratoire d'Acoustique de Métrologie et d'Instrumentation, 38 rue des 36 Ponts, 31400 Toulouse, France

\begin{abstract}
Résumé : Cet article présente l'utilisation d'un réseau de neurones multicouche pour la reconnaissance automatique de signaux acoustiques. Les signaux considérés sont obtenus en environnement bruyant, ils sont non stationnaires, non reproductibles entre deux événements et sont de date d'apparition aléatoire. Suite à deux méthodes de modélisation, le réseau de neurones est utilisé comme classificateur supervisé. Ses performances seront données en terme de probabilités de détection et de fausse alarme.

Abstract : This article presents the use of a neural multi-layer network for automatic recognition of acoustic signals. The considered signals are obtained in a noisy environment, they are nonstationary, non-reproducible between two events and have a random occurrence date. Further to modeling methods, the neural network is used as a supervised classifier. Its performance will be given in terms of detection and false alarm probabilities.
\end{abstract}

\section{I - INTRODUCTION}

Dans le cadre de la reconnaissance et de la classification de signatures acoustiques, nous avons à reconnaître un signal d'origine biologique.

Pour résoudre ce problème, nous avons d'abord cherché à modéliser le signal avant d'effectuer une classification. L'étude temps fréquence du signal a permis de retenir deux méthodes de modélisation différentes, une en valeur moyenne d'énergie par bande de fréquence et l'autre par modélisation autorégressive. La classification, quand à elle, est effectuée par un réseau de neurones multicouche. Celui-ci commence par adapter ces divers paramètres au problème grâce à des couples de points entrées-sorties choisis. Quand les paramètres du réseau sont stables, on l'utilise alors comme classificateur.

Nous allons donc décrire succinctement le fonctionnement d'un réseau multicouche, puis présenter les signaux que l'on a traité ainsi que les résultats obtenus avant de conclure.

\section{2 - PRESENTATION DU RESEAU NEURO-MIMETIQUE MULTICOUCHE}

Nous avons utilisé un réseau neuro-mimétique [1][2] à trois couches qui est composé d'une couche d'entrée dont les cellules, par convention, se contentent de transmettre les entrées et de deux couches successives de cellules élémentaires encore appelées neurones formels interconnectés entre eux (voir figure 1). Les connexions sont à sens unique de l'entrée vers la sortie, le réseau est donc non bouclé. La couche intermédiaire, dont les sorties ne sont pas directement accessibles, est appelée la couche cachée. Chaque neurone formel réalise la fonction : $\mathrm{s}=\mathrm{f}\left(\sum_{\mathrm{i}} \mathrm{w}_{\mathrm{ij}} \mathrm{e}_{\mathrm{j}}\right)$ où $\left\{\mathrm{e}_{\mathrm{j}}\right\}$ est l'ensemble des entrées de la cellule, $\left\{\mathrm{w}_{\mathrm{ij}}\right\}$ est l'ensemble des poids synaptiques des connexions, $f$ est la fonction d'activation (c'est une fonction continue discriminante fixée : une sigmoïde dans notre cas) et s est la sortie unique du neurone formel.

L'utilisation d'un tel réseau de neurones (RN) se décompose en deux parties : la phase d'apprentissage et la phase de généralisation. Lors de la première phase, on effectue un apprentissage supervisé pour adapter les poids des connexions au problème posé. Pour cela on utilise un algorithme du gradient dont les principales équations pour un réseau à deux couches de poids modifiables sont : 
Soit une base d'apprentissage $\left\{X^{(a)}, Y^{(a)}, 1 \leq a \leq N\right\}$

- $X^{(a)}=\left(X_{1}^{(a)} \ldots X_{n}^{(a)}\right)$ est vecteur d'entrée,

' - $Y^{(a)}=\left(Y_{1}^{(a)} \ldots Y_{m}^{(a)}\right)$ est le vecteur de sortie correspondant,

$-S^{(a)}=\left(S_{1}^{(a)} \ldots S_{m}^{(a)}\right)$ un vecteur de sortie effectivement obtenu,

- w est la matrice des poids de la couche intermédiaire,

- W est la matrice des poids de la couche de sortie,

- $\mathrm{f}$ est la fonction d'activation d'un neurone et $\mathrm{f}$ ' sa dérivée

On cherche à minimiser l'erreur obtenue suite à un passage complet de la base d'apprentissage : $\mathrm{E}=\sum_{\mathrm{a}} \mathrm{E}^{(\mathrm{a})}$ ce qui revient à minimiser l'erreur globale pour chaque élément de la base d'apprentissage d'où $E^{(a)}=\frac{1}{2} \sum_{k}\left(S_{k}^{(a)}-Y_{k}^{(a)}\right)^{2}$.

On modifie les poids du réseau dans le sens rétrograde en utilisant une méthode du gradient de pas $\mu$, ce qui donne : $\Delta \mathrm{W}_{\mathrm{ij}}=-\frac{\mu \partial \mathrm{E}^{(\mathrm{a})}}{\partial \mathrm{W}_{\mathrm{ij}}}$. La mise à jour des poids est faite à chaque présentation d'un élément de la base d'apprentissage ; c'est un algorithme de rétro-propagation du gradient stochastique. Tous calculs fait on obtient les relations :

- pour les poids de la couche de sortie : $\Delta \mathrm{W}_{\mathrm{kj}}=\mu\left(\mathrm{S}_{\mathrm{k}}^{(\mathrm{a})}-\mathrm{Y}_{\mathrm{k}}^{(\mathrm{a})}\right) \mathrm{f}^{\prime}\left(\sum_{\mathrm{j}} \mathrm{W}_{\mathrm{kj}} \mathrm{f}\left(\sum_{\mathrm{i}} \mathrm{w}_{\mathrm{ji}} \mathrm{X}_{\mathrm{i}}^{(\mathrm{a})}\right)\right) \mathrm{f}\left(\sum_{\mathrm{i}} \mathrm{w}_{\mathrm{ji}} \mathrm{X}_{\mathrm{i}}^{(\mathrm{a})}\right)$

- pour les poids de la couche intermédiaire :

$$
\Delta \mathrm{w}_{\mathrm{ji}}=\mu \sum_{\mathrm{k}}\left[\left(\mathrm{S}_{\mathrm{k}}^{(\mathrm{a})}-\mathrm{Y}_{\mathrm{k}}^{(\mathrm{a})}\right) \mathrm{W}_{\mathrm{kj}} \mathrm{f}^{\prime}\left(\sum_{\mathrm{j}} \mathrm{W}_{\mathrm{kj}} \mathrm{f}\left(\sum_{\mathrm{i}} \mathrm{w}_{\mathrm{ji}} \mathrm{X}_{\mathrm{i}}^{(\mathrm{a})}\right)\right)\right] \mathrm{f}^{\prime}\left(\sum_{\mathrm{i}} \mathrm{w}_{\mathrm{ji}} \mathrm{X}_{\mathrm{i}}^{(\mathrm{a})}\right) \mathrm{X}_{\mathrm{i}}^{(\mathrm{a})}
$$

les indices sont relatifs aux différentes couches : i pour la couche d'entrée, j pour l'intermédiaire et $\mathrm{k}$ pour celle de sortie.

Nous avons utilisé des biais pour chaque neurone formel, ce qui n'apparaît pas les équations. Mais, comme les biais ont exactement le même comportement que les poids, il suffit de considérer que l'entrée correspondante vaut toujours un.

Le nombre de neurones par couche sera adapté à chaque méthode de modélisation. Pour la couche d'entrée, il y a un neurone par paramètre retenu pour décrire les individus. Au niveau de la couche cachée on adapte le nombre de neurones en fonction des résultats. Quant à la couche de sortie, on a pris un neurone par classe $\left(\mathrm{C}_{\mathrm{i}}\right)$ avec le codage suivant : $\mathrm{X}^{(\mathrm{a})} \in \mathrm{C}_{\mathrm{i}} \Leftrightarrow \mathrm{S}_{\mathrm{k}}^{(\mathrm{a})}=\delta_{\mathrm{ik}}$ où $\delta_{i k}=0$ si $\mathrm{i}=\mathrm{k} \delta_{i k}=0$ sinon. Quand un individu appartient à deux classes différentes ou à aucune d'entre elles, il est considéré comme hors classe.

Quand les poids du RN sont figés, on peut utiliser le réseau en phase de généralisation, on lui présente des individus inconnus qu'il doit classer.

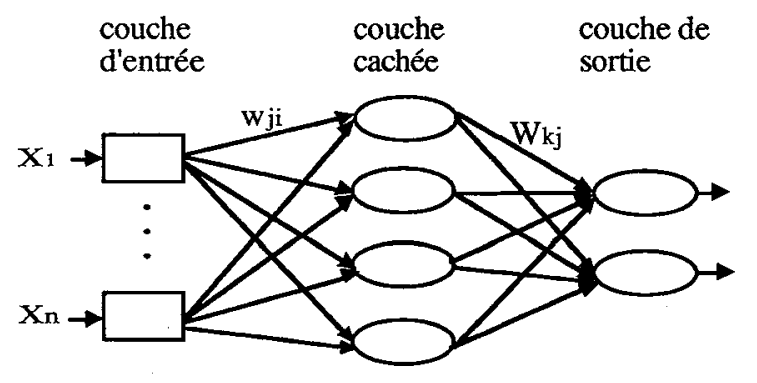

figure 1 : représentation d'un réseau de neurones multicouche 


\section{3 - DESCRIPTION DES SIGNAUX}

Le signal que nous avons à reconnaître de manière automatique est le son produit par l'alose, un poisson migrateur de la Garonne, lors de sa ponte à la surface de l'eau [3]. Le comptage du nombre de ponte a pour but d'effectuer le recensement de la population de ces animaux.

Les signaux sont recueillis par l'intermédiaire d'un microphone disposé sur les berges du fleuve en période de fraie. Les sons provoqués par les poissons sont difficiles à reconnaître car leur date d'apparition est aléatoire, ils sont non stationnaires et non reproductibles entre deux événements. De plus, de par le mode de recueil des signaux, ils sont souvent bruités par des bruits de véhicules routiers, d'oiseaux, de grenouilles...

Une représentation temporelle du signal est donnée sur la figure 2.

Une étude temps fréquence du signal produit par l'alose (figure 3) a permis de déterminer un spectre moyen qui est à la base d'une des techniques de modélisation retenue. Celle-ci consiste en l'extraction de l'énergie moyenne dans trois bandes de fréquence, ce qui nous donne des individus définis par trois paramètres. La seconde méthode de modélisation que nous avons utilisée consiste à calculer les premiers coefficients du modèle auto-régressif (AR) d'ordre sept du signal. Dans ce cas, on obtient des individus représentés par huit paramètres, les sept coefficients du modèle et l'énergie du bruit d'entrée du modèle.

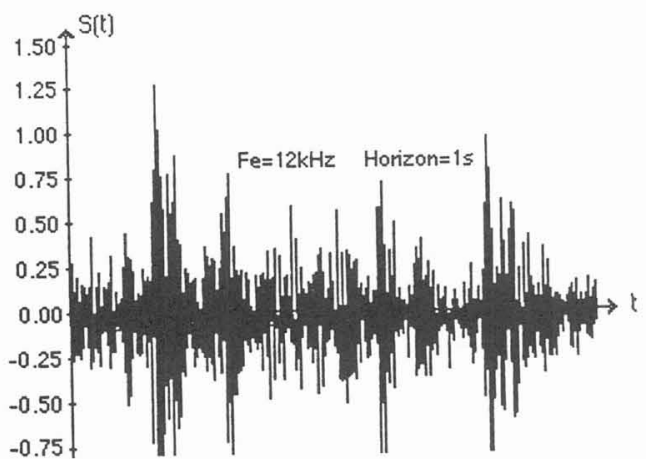

figure 2 : représentation temporelle du signal produit par l'alose

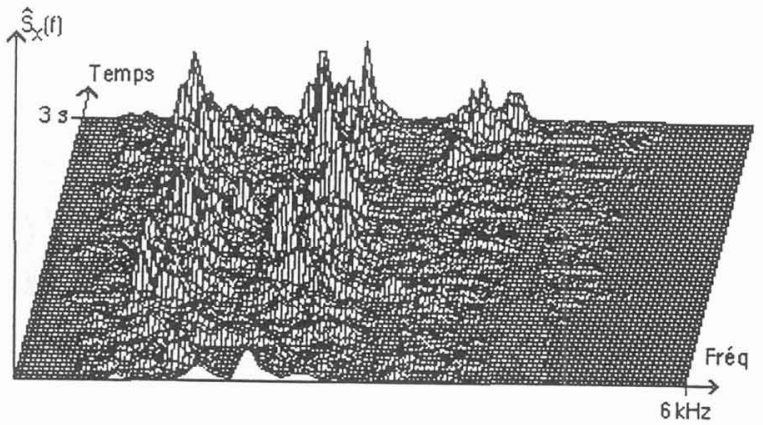

figure 3 : évolution spectrale du signal produit part l'alose

\section{4 - PRESENTATION DES RESULTATS}

Relativement à une classe $\mathrm{i}$, l'univers des événements se divise en deux parties. Celle contenant les individus de la classe $\left(x_{i}\right)$ et celle ne les contenant pas $\left(\overline{x_{i}}\right)$. Par contre, le classificateur va créer deux zones légèrement différentes. L'une où il a déterminé la présence de la classe $\left(\mathrm{dx}_{\mathbf{i}}\right)$ et l'autre où il ne l'a pas déterminée $\left(d \overline{x_{i}}\right)$. Donc si on pose :

- Nbr_dx $x_{i} x_{i}=$ le nombre d'individus affectés à la classe i sachant qu'ils appartiennent à cette classe,

- Nbr_d $d \overline{x_{i}}-x_{i}=$ le nombre d'individus non affectés à la classe i sachant qu'ils appartiennent à cette classe,

- Nbr_Total= le nombre total d'individus classés.

On obtient :

$$
\begin{array}{lr}
-P\left(d x_{i} \cap x\right)=\frac{N b r_{-} d x_{i}-x_{i}}{N b r_{-} T o t a l} & -P\left(x_{i}\right)=\frac{N b r_{-} d x_{i} x_{i}+N b r_{-} d \overline{x_{i}}-x_{i}}{N b r_{-} T o t a l} \\
-P\left(d \overline{x_{i}} \cap x_{i}\right)=\frac{N b r_{-} d \overline{x_{i}}-x_{i}}{N b r_{-} \text {Total }} & -P\left(\overline{x_{i}}\right)=\frac{N b r_{-} d x_{i} \overline{x_{i}}+N b r_{-} d \overline{x_{i}}-\overline{x_{i}}}{N b r_{-} \text {Total }}
\end{array}
$$

Bien que :

taux de réussite: $T_{R}=P\left(d x_{i} \cap x_{i}\right)+P\left(d \overline{x_{i}} \cap \overline{x_{i}}\right)$ et taux d'échec: $T_{E}=P\left(d x_{i} \cap \overline{x_{i}}\right)+P\left(d \overline{x_{i}} \cap x_{i}\right)$

ces résultats risquent de masquer ce qui nous intéresse dans le cas où les probabilités $p\left(x_{i}\right)$ et $p\left(\overline{x_{i}}\right)$ sont très différentes. C'est pourquoi il est préférable de considérer les probabilités conditionnelles :

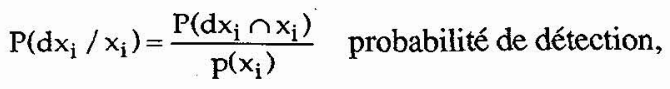




$$
\begin{aligned}
& \mathrm{P}\left(\mathrm{dx_{i }} / \overline{x_{i}}\right)=\frac{P\left(d x_{i} \cap \overline{x_{i}}\right)}{p\left(\overline{x_{i}}\right)} \text { probabilité de fausse alarme, } \\
& \mathrm{P}\left(\overline{d x_{i}} / x_{i}\right)=\frac{P\left(d \overline{x_{i}} \cap x_{i}\right)}{\mathrm{p}\left(x_{i}\right)} \quad \text { probabilité de non détection, } \mathrm{P}\left(\bar{d} \overline{x_{i}} / \overline{x_{i}}\right)=\frac{P\left(d \overline{x_{i}} \cap \overline{x_{i}}\right)}{\mathrm{p}\left(\overline{x_{i}}\right)}
\end{aligned}
$$

En fait deux de ces probabilités suffisent à résumer les performances du classificateur car on a : $\mathrm{P}\left(\mathrm{dx}_{\mathrm{j}} / \mathrm{xi}_{\mathbf{i}}\right)+\mathrm{P}\left(\mathrm{d} \overline{\mathrm{x}_{\mathbf{i}}} / \mathrm{x}_{\mathbf{i}}\right)=1$ et $\mathrm{P}\left(\mathrm{dx}_{\mathrm{i}} / \overline{\mathrm{x}_{\mathbf{i}}}\right)+\mathrm{P}\left(\mathrm{d} \overline{\mathrm{x}_{\mathbf{i}}} / \overline{\mathrm{x}_{\mathbf{i}}}\right)=1$

Nous avons utilisé trois fichiers différents, un contenant les signaux de l'alose bien distincts des autres bruits (fichiers en 1) un autre contenant des signaux d'alose qui sont très bruités (fichiers en 2). Ces deux fichiers seront utilisés lors de la phase de généralisation. Le troisième fichier (en 12) est la base d'apprentissage qui est composé à moitié de signaux d'alose bruités ou non et de signaux bruit. On distinguera également la modélisation en énergie (fichiers en EN) de la modélisation AR (fichiers en AR).

Pour les fichiers en $1: \mathrm{P}($ Alose $)=50 \% \mathrm{P}($ Bruit $)=10 \%$,

pour les fichiers en $2: \mathrm{P}($ Alose $)=12 \% \mathrm{P}($ Bruit $)=65 \%$.

tableau $1:$ les différentes probabilités obtenus en fonction du fichier considéré

\begin{tabular}{|l|c|c|c||c|c|c|}
\cline { 2 - 7 } \multicolumn{1}{c|}{} & EN12 & E1 & E2 & AR12 & AR1 & AR2 \\
\hline $\mathrm{T}_{\mathrm{R}}$ (Bruit) & 86,5 & 86,3 & 86,4 & 84,8 & 82,1 & 81,6 \\
$\mathrm{~T}_{\mathrm{R}}$ (Alose) & 86,0 & 86,3 & 86,3 & 84,7 & 82,0 & 81,8 \\
\hline $\mathrm{P}(\mathrm{dB} / \mathrm{B})$ & 90,0 & 89,9 & 86,0 & 89,5 & 88,6 & 80,7 \\
$\mathrm{P}(\mathrm{dAl} / \mathrm{Al})$ & 82,0 & 82,7 & 87,2 & 79,7 & 75,4 & 87,3 \\
\hline $\mathrm{P}(\mathrm{dB} / \overline{\mathrm{B}})$ & 17,0 & 17,3 & 11,0 & 20,0 & 24,3 & 12,6 \\
$\mathrm{P}(\mathrm{dAl} / \overline{\mathrm{Al}})$ & 10,0 & 9,9 & 13,9 & 10,4 & 11,3 & 19,1 \\
\hline
\end{tabular}

Ces résultats sont un compromis entre la probabilité de détection et la probabilité de fausse alarme car l'amélioration de l'une ce fait au détriment de l'autre.

\section{5 - CONCLUSIONS}

Le réseau de neurones multicouche est un classificateur robuste qui a l'avantage de ne pas nécessiter de prétraitement des paramètres. Car, pour obtenir des résultats comparables avec l'algorithme des centres mobiles [4] il faut d'abord effectuer une très longue étape de choix et de mise en forme des paramètres.

Les résultats obtenus avec le réseau montrent l'intérêt, dans notre cas, de la modélisation en énergie par rapport à la modélisation $\mathrm{AR}$. En effet, la première méthode effectue une moyenne qui rend l'ensemble du système plus robuste aux contraintes dues à l'environnement bruyant et à la non reproductibilité entres deux événements des signaux étudiés.

Avant de conclure sur les capacités du réseau multicouche pour la reconnaissance de signatures acoustiques, les performances doivent être évalués sur d'autres classes de signaux. De plus, il serait intéressant de déterminer l'influence des divers paramètres fournis en entrée.

[1] J.M. NICOLAS, A. LEMER \& D. LEGITIMUS

Identification automatique de bruits impulsifs en acoustique sous-marine par réseaux multicouches.

Proc. Neuro Nîmes 1989, p.269-278.

[2] P COMON

Classification supervisée par réseaux multicouches.

Traitement du signal, vol. $8, \mathrm{n}^{\circ} 61991$, p.387-407

[3] J.F. TROUILHET

Etude et réalisation d'un dispositif de reconnaissance automatique de signature acoustique

Thèse de doctorat de l'INP Toulouse Juin 1992

[4] J.F. TROUILHET, I. COUSTAUX, J.P. GUILHOT

Reconnaissance des formes dans le plan temps fréquence par modélisation paramétrique.

GRETSI Sept. 93, p.25-28 